\title{
The Interplay between Radiation Pressure and the Photoelectric Instability in Optically Thin Disks of Gas and Dust
}

\author{
Alexander J. W. Richert ${ }^{1,2}$ (D), Wladimir Lyra ${ }^{3,4,5}$ (D), and Marc J. Kuchner ${ }^{2}$ (D) \\ ${ }^{1}$ Department of Astronomy \& Astrophysics, Penn State University, 525 Davey Lab, University Park, PA 16802, USA; alexrichert@gmail.com \\ ${ }^{2}$ Exoplanets and Stellar Astrophysics Laboratory, NASA Goddard Space Flight Center, 8800 Greenbelt Road, Greenbelt, MD 20771, USA \\ Department of Physics and Astronomy, California State University Northridge, 18111 Nordhoff Street, Northridge, CA 91330, USA \\ ${ }^{4}$ Jet Propulsion Laboratory, California Institute of Technology, 4800 Oak Grove Drive, Pasadena, CA 91109, USA \\ ${ }^{5}$ Division of Geological \& Planetary Sciences, California Institute of Technology, 1200 East California Boulevard MC 150-21, Pasadena, CA 91125, USA \\ Received 2017 September 12; revised 2018 February 1; accepted 2018 February 5; published 2018 March 22
}

\begin{abstract}
In optically thin disks, dust grains are photoelectrically stripped of electrons by starlight, heating nearby gas and possibly creating a dust clumping instability - the photoelectric instability (PeI) - that significantly alters global disk structure. In the current work, we use the Pencil Code to perform the first numerical models of the PeI that include stellar radiation pressure on dust grains in order to explore the parameter regime in which the instability operates. In some models with low gas and dust surface densities, we see a variety of dust structures, including sharp concentric rings. In the most gas- and dust-rich models, nonaxisymmetric clumps, arcs, and spiral arms emerge that represent dust surface density enhancements of factors of $\sim 5-20$. In one high gas surface density model, we include a large, low-order gas viscosity and find that it observably smooths the structures that form in the gas and dust, suggesting that resolved images of a given disk may be useful for deriving constraints on the effective viscosity of its gas. Our models show that radiation pressure does not preclude the formation of complex structure from the PeI, but the qualitative manifestation of the PeI depends strongly on the parameters of the system. The PeI may provide an explanation for unusual disk morphologies, such as the moving blobs of the AU Mic disk, the asymmetric dust distribution of the 49 Ceti disk, and the rings and arcs found in the HD 141569A disk.
\end{abstract}

Key words: hydrodynamics - instabilities - methods: numerical - radiation: dynamics - zodiacal dust

\section{Introduction}

Circumstellar disks play a key role in testing theories of planet formation and evolution, revealing the physical and chemical environment of planet-forming systems, including providing constraints on the properties of nascent planets. Resolved images of protoplanetary disks, transitional disks, and debris disks show a variety of complex morphologies, including cavities, gaps, and rings (Debes et al. 2013; Wahhaj et al. 2014; ALMA Partnership et al. 2015; Currie et al. 2015; Follette et al. 2015; van Boekel et al. 2017), as well crescentshaped structures, arcs, and spiral arms (Grady et al. 2013; van der Marel et al. 2013; Biller et al. 2015; Perrot et al. 2016; Follette et al. 2017). These disk structures are frequently attributed to gravitational perturbation by unseen embedded planets (e.g., Kuchner \& Holman 2003; Nesvold \& Kuchner 2015; Richert et al. 2015; Dong \& Dawson 2016; Dipierro \& Laibe 2017; Dong \& Fung 2017; Dong et al. 2017).

The possibility of comparable masses of gas and dust in any given optically thin disk raises the possibility of hydrodynamical interactions that will give rise to features like gaps, rings, and clumps that are frequently attributed to gravitational perturbation by an unseen embedded planet (Klahr \& Lin 2005; Besla \& Wu 2007; Lyra \& Kuchner 2013). In optically thin disks, stellar far-ultraviolet photons whose energies exceed the work function of the dust grains (a few eV; Besla \& Wu 2007) photoelectrically eject electrons, which in turn heat nearby gas. Klahr \& Lin (2005) and Besla \& Wu (2007) propose that this leads to a clumping instability-the photoelectric instability (PeI) - wherein a heating of the gas by dust grains creates a local pressure maximum, which then traps more dust, which further heats the gas, and so on. Klahr \& Lin (2005) model the system in $1 \mathrm{D}$, find the instability, and extrapolate from the 1D results to suggest that in $2 \mathrm{D}$, the photoelectric instability will generate ring structures that are similar to those observed in disks like the one around HR 4796A. Lyra \& Kuchner (2013) model the system hydrodynamically with $2 \mathrm{D}$ global and 3D local simulations and find that rings are not formed unless the acceleration of gas due to aerodynamic drag backreaction from the dust is considered. When that component is ignored, power concentrates in high azimuthal wavenumbers and only clumps are formed. When the action of the dust on the gas is considered, rings and incomplete arcs are seen to form in the dust distribution.

Other findings of Lyra \& Kuchner (2013) are that (1) linear instability exists only for the dust-to-gas ratio $\epsilon_{\mathrm{d} / \mathrm{g}}<1$, with maximum growth rate at $\epsilon_{\mathrm{d} / \mathrm{g}} \approx 0.2 ;(2)$ nonlinear instability is observed for $\epsilon_{\mathrm{d} / \mathrm{g}}=1$; (3) linear instability only exists if photoelectric heating is the dominant heating source; (4) the photoelectric instability supersedes the streaming instability when the conditions for both are present; and (5) the particular mode for which gas and dust velocity are equal, thus canceling the drag force and backreaction, executes free oscillations, which are seen as a small, but finite, eccentricity $(\approx 0.03)$.

The photoelectric instability may provide an explanation for a number of observed systems with unusual morphologies. Scattered light images of the AU Mic disk, an edge-on system, reveal radially moving blobs not seen at longer wavelengths. The disk around HIP 73145 contains concentric rings in scattered light images (which reveal small grains), while larger grains, observed at ALMA wavelengths, are distributed more compactly around the central star (Feldt et al. 2017). For both of these systems, the differing behavior of small and large 
grains is not readily explained by a planetary perturber, which will exert equal graviational pull on grains of all sizes. The edge-on disk around 49 Ceti is known to be gas rich, though the total mass remains poorly constrained (Hughes et al. 2017). Hughes et al. (2017) identify an asymmetric structure in the disk that is consistent with a warp or spiral arm, finding no such features in two resolved gas-poor disks. The HD 141569A transition disk contains rings and arclets of small grains (Perrot et al. 2016). The findings of Klahr \& Lin (2005), Besla \& Wu (2007), and Lyra \& Kuchner (2013) also raise the question of whether the presence of gas plays a role in the formation of the sharp dust rings seen in scattered light images of disks, such as those around HR 4796A (Milli et al. 2017) and Fomalhaut (Kalas et al. 2008).

Although they are novel, the hydrodynamical models of Lyra \& Kuchner (2013) do not include radiation pressure from the central star on dust grains, which, even around low-mass stars, will put small grains on highly eccentric orbits, and in some cases will blow them out of the system. The ability of the photoelectric instability to explain the morphologies of optically thin disks depends vitally on whether it can operate in the presence of stellar radiation pressure on dust grains.

In this work, we conduct hydrodynamical simulations of optically thin disks that include both dust-gas photoelectric heating and radiation pressure on dust grains that span a range of sizes. In Section 2, we provide an analytical discussion of the role of grain size, with respect to the emergence of hydrodynamical instabilities. Equations solved and initial conditions are discussed in Section 3. Results are discussed in Section 4, while further conclusions and implications for future work are discussed in Section 6.

\section{The Role of Grain Size}

Stellar radiation pressure accelerates dust grains away from the host star in optically thin disks, potentially inhibiting the formation of the clumps, arcs, and rings seen in the models of Lyra \& Kuchner (2013). For spherical grains, the radiation pressure strength $\beta$, defined as the ratio of the radiation force to the gravitational force, depends on host star mass $M_{\star}$, host star luminosity $L_{\star}$, grain radius $a$, and dust material density $\rho_{\text {mat }}$ (Burns et al. 1979; Krivov 2010), such that

$$
\beta \simeq 0.574 \frac{M_{\star}}{M_{\odot}}\left(\frac{L_{\star}}{L_{\odot}}\right)^{-1}\left(\frac{a}{1 \mu \mathrm{m}}\right)^{-1}\left(\frac{\rho_{\mathrm{mat}}}{1 \mathrm{~g} \mathrm{~cm}^{-3}}\right)^{-1}
$$

When a dust grain is created on a Keplerian orbit, radiation pressure places it on an eccentric orbit where eccentricity $e=\beta /(1-\beta)$ (Burns et al. 1979; Strubbe \& Chiang 2006). A grain with $\beta=\frac{1}{2}$ receives a radiation force equal to half the gravitational force, causing it to become unbound $(e=1)$. The models produced in this work will help to determine whether non-zero orbital eccentricities of dust grains affect the onset of clumping instabilities.

Clumping due to the photoelectric instability depends on aerodynamic drag. Lyra \& Kuchner (2013) find that the instability is robust for this variable, meaning that grains of longer stopping time simply take longer to respond to the pressure maximum and concentrate. Yet, one can imagine that if other dynamical processes are modifying the state of the gas at timescales shorter than the stopping time of the grains, clumping by the photoelectric instability may be disrupted. In

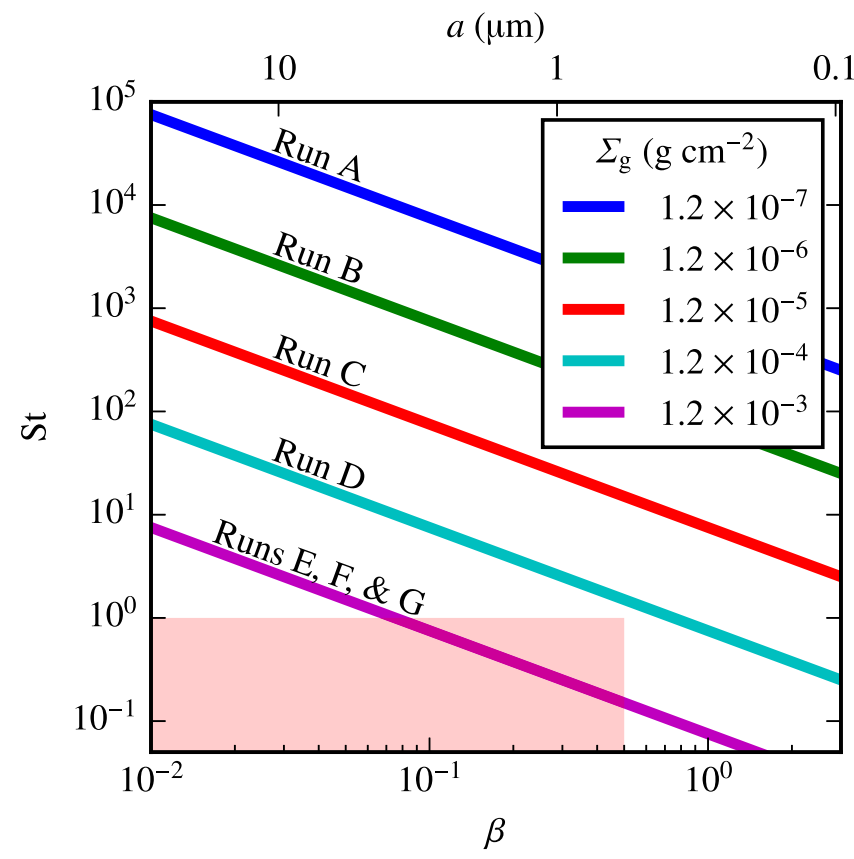

Figure 1. Dimensionless drag stopping time St as a function of $\beta$ for several values of gas surface density $\Sigma_{\mathrm{g}}$, assuming a solar-type star. The pink shaded area indicates bound grains $\left(\beta<\frac{1}{2}\right)$ with short stopping times $(\mathrm{St}<1)$.

other words, although linear growth is present, the saturated state may be quite different for small and big grains. Given the stopping time $\tau_{\mathrm{f}}$, a nondimensional stopping time can be constructed, also known as Stokes number, $\mathrm{St} \equiv \tau_{\mathrm{f}} \Omega \mathrm{K}$, where $\Omega_{\mathrm{K}}$ is the Keplerian frequency. For a thin disk,

$$
\mathrm{St} \approx \frac{\pi a \rho_{\mathrm{mat}}}{2 \Sigma_{\mathrm{g}}}
$$

where $\Sigma_{\mathrm{g}}$ is the surface density of the gas. Grain size therefore seems likely to play a dual role in the development of dust clumping instabilities in optically thin disks, which require grains small enough to be susceptible to aerodynamic drag, but large enough to remain bound to the star, preferably on a loweccentricity orbit.

The relationship between $\mathrm{St}$ and $\beta$ is easily specified for spherical grains in a thin disk based on Equations (1) and (2), such that

$$
\mathrm{St}=\frac{1}{\beta}\left(\frac{\Sigma_{\mathrm{g}}}{9 \times 10^{-5} \mathrm{~g} \mathrm{~cm}^{-2}}\right)^{-1} \frac{L_{\star}}{L_{\odot}}\left(\frac{M_{\star}}{M_{\odot}}\right)^{-1} .
$$

In Figure 1, we show St as a function of $\beta$ for a solar-type star for several values of $\Sigma_{g}$. The gas surface density values shown are a few orders of magnitude below the densities where the optical thickness of the gas will impede both the photoelectric stripping of dust and the radiation pressure.

As a priori it would seem that a substantial level of gas is required in order to rapidly create dust clumping instabilities in the presence of radiation pressure, while larger grains may be able to generate such instabilities over longer timescales (Lyra \& Kuchner 2013). Yet, clumping by the PeI in the presence of radiation pressure may require grains of Stokes number near unity. A shorter PeI onset timescale would allow the PeI to operate in disks spanning a large range of dust production rates (which remain poorly constrained in observed systems). 


\section{Methods}

\subsection{Equations}

In this work, we perform 2D global simulations of gasbearing optically thin circumstellar disks using the Pencil Code, which is a high-order finite difference hydrodynamics code (Brandenburg \& Dobler 2002). Both the gas and dust are calculated in Cartesian coordinates. ${ }^{6}$ The code evolves the gas according to the continuity equation,

$$
\frac{D \Sigma_{\mathrm{g}}}{D t}=-\Sigma_{\mathrm{g}} \boldsymbol{\nabla} \cdot \boldsymbol{u}
$$

and the equation of motion,

$$
\frac{D \boldsymbol{u}}{D t}=-\frac{1}{\Sigma_{\mathrm{g}}} \nabla P-\nabla \Phi_{\star}-\frac{\Sigma_{\mathrm{d}}}{\Sigma_{\mathrm{g}}} f_{\mathrm{d}},
$$

where $\Sigma_{\mathrm{g}}$ and $\Sigma_{\mathrm{d}}$ are the gas and dust surface densities, and $\boldsymbol{u}$ and $P$ are the velocity and pressure of the gas. $\Phi_{\star}$ represents the Newtonian gravitational potential of the star. The dust drag term $f_{\mathrm{d}}$ is defined below. The mean-free paths associated with the gas densities we model (Section 3.2) are much smaller than the scale heights that are under consideration for a range of gas compositions (including an $\mathrm{H}_{2}$-dominated disk), making a fluid treatment of the gas appropriate.

We model the dust using 400,000 Lagrangian superparticles of equal mass, except for two runs where the total dust mass is increased in part by doubling the number of superparticles to 800,000 (see Section 3.2). Each superparticle contains subparticles of one physical radius $a$ between 0.1 and $10 \mu \mathrm{m}$, and all subparticles have a constant material density of $\rho_{\text {mat }}=2 \mathrm{~g} \mathrm{~cm}^{-3}$, or the approximate material density of silicate grains. This yields a total dust mass of $\sim 0.01 M_{\oplus}$.

The overall grain size distribution follows the standard Dohnanyi (1969) $q=-3.5$ power law. The use of superparticles of the same mass automatically contributes a dependence with $q=-3$ (superparticles with smaller grains will contain more particles); grain sizes associated with superparticles follow a $q=-0.5$ dependence in order to yield a $q=-3.5$ size distribution overall. This scheme prevents numerical issues associated with consolidating large grains into a small numbers of very massive superparticles (which, especially when modeling photoelectric heating, can crash the code).

In all simulations, superparticles are inserted in a birth ring positioned at $100 \mathrm{au}$ from the central star; the birth ring is axisymmetric and has a radial Gaussian profile in order to avoid an artificial sharp edge in the dust distribution. For 7 of our 8 runs, this Gaussian profile has width $\sigma_{\mathrm{BR}}=10 \mathrm{au}$, while for the eighth, $\sigma_{\mathrm{BR}}=20 \mathrm{au}$ in order to ensure that the results seen in the other simulations are not peculiar to a narrow birth ring. Superparticles are at first inserted gradually over the course of 50 orbits in order to avoid discontinuities and ensure that superparticles span a range of orbital phases, but then are inserted throughout the simulation so as to yield a constant number of superparticles and therefore a constant total mass of the disk. That is to say, when a superparticle crosses the inner $(50 \mathrm{au})$ or outer $(800 \mathrm{au})$ boundary of the disk, another

\footnotetext{
6 In our preliminary runs, we identified a bug in the Pencil Code that affected the calculation of azimuthal particle accelerations in polar coordinates. The bug has since been fixed and does not seem to have adversely affected previous works using that part of the code.
}

superparticle is inserted in the birth ring with a new grain size chosen at random according to the $q=-3.5$ distribution.

The dynamical equation for each dust superparticle with velocity $\boldsymbol{v}$ depends on the effective gravitational potential $\Phi_{\mathrm{eff}}$ and the gas drag term $f_{\mathrm{d}}$,

$$
\frac{d v}{d t}=-\Phi_{\mathrm{eff}}+f_{\mathrm{d}} .
$$

The expression for the drag acceleration is given by

$$
f_{\mathrm{d}}=-\left(\frac{2 \Omega_{\mathrm{K}} \Sigma_{\mathrm{g}} C_{D}}{\pi a \rho_{\text {mat }}}\right) \Delta \boldsymbol{v},
$$

where $\Omega_{\mathrm{K}}$ is the Keplerian orbital frequency at a given orbital radius, and gas-dust velocity differential $\Delta \boldsymbol{v}=\boldsymbol{v}-\boldsymbol{u}$. The low gas densities of debris disks imply very large mean-free paths compared with dust grain radii (i.e., $\lambda \gg a$ ), hence the drag coefficient $C_{D}$ is calculated for the Epstein regime as a function of sound speed $c_{s}$ such that

$$
C_{D}=\sqrt{1+\frac{9 \pi}{128}\left(|\Delta v| / c_{S}\right)^{2}} .
$$

The effective gravitational potential term $\Phi_{\text {eff }}$ incorporates radiation pressure on the dust, such that

$$
\Phi_{\text {eff }}=\frac{G M_{\star}(1-\beta)}{r^{2}},
$$

where for each superparticle, the ratio of the radiation pressure force to gravitational force is $\beta=\beta_{\text {ref }} / a$. We calculate reference radiation pressure strength $\beta_{\text {ref }}$ for a solar-type star according to the prescription of Burns et al. (1979), such that $\beta \approx 0.2$ for a $1 \mu \mathrm{m}$ grain with a density of $2 \mathrm{~g} \mathrm{~cm}^{-3}$. Our grain size range corresponds with radiation pressure strengths $0.03<\beta<3$, which in turn corresponds with eccentricities ranging from near-circular to completely unbound orbits, where an unbound orbit $(e \geqslant 1)$ has $\beta>0.5$, corresponding with a grain size of $a<0.57 \mu \mathrm{m}$.

Given that the photoelectric heating time is small compared with the dynamical time (Besla \& Wu 2007; Lyra \& Kuchner 2013), we adopt the modified equation of the state of Lyra \& Kuchner (2013) that implements instantaneous heating of gas by dust. The gas pressure $P$ is assumed to be proportional to the dust density $\Sigma_{\mathrm{d}}$, such that

$$
\nabla P=\frac{\Theta c_{s 0}^{2}}{\gamma \Sigma_{\mathrm{g}, 0}}\left(\Sigma_{\mathrm{g}} \nabla \Sigma_{\mathrm{d}}+\Sigma_{\mathrm{d}} \nabla \Sigma_{\mathrm{g}}\right) .
$$

$\Theta$ is a dimensionless parameter that sets the pressure contribution of photoelectric heating compared to the background temperature of the gas (Lyra \& Kuchner 2013), which is itself specified by the reference sound speed $c_{s 0}=0.05$ in code units, corresponding with a scale height of 0.05 , which is assumed for both the gas and dust. The value of $\Sigma_{\mathrm{d}}$ is calculated by interpolating particle positions onto the grid using a triangular-shaped cloud particle mesh scheme (Eastwood \& Hockney 1974). We assume that the gas is locally isothermal, which is appropriate for the short cooling times expected in debris disks (Lyra \& Kuchner 2013).

The high-order scheme used by the Pencil Code leads to little numerical dissipation, therefore we apply sixth-order hyperdissipation terms to the right-hand side of Equations (4) 
Table 1

Run Parameters

\begin{tabular}{|c|c|c|c|c|c|c|c|c|c|c|}
\hline Run & $\begin{array}{c}\Sigma_{\mathrm{g}, 0} \\
\left(\mathrm{~g} \mathrm{~cm}^{-2}\right)\end{array}$ & $\begin{array}{c}\text { Grid } \\
\text { Resolution }\end{array}$ & $\begin{array}{c}\text { Number of Dust } \\
\text { Superparticles }\end{array}$ & Total & $\begin{array}{l}\text { Dust Mass } \\
\left(M_{\oplus}\right)\end{array}$ & $\alpha$ & $\begin{array}{l}\sigma_{\mathrm{BR}} \\
(\mathrm{au})\end{array}$ & $\min (\mathrm{St})$ & $\begin{array}{c}\left\langle\epsilon_{\mathrm{d} / \mathrm{g}}\right\rangle \\
(400 \text { Orbits) }\end{array}$ & $\tilde{\tau}$ \\
\hline A & $1.2 \times 10^{-7}$ & $1008^{2}$ & $4 \times 10^{5}$ & 9 & $\times 10^{-3}$ & $\cdots$ & 10 & $2.6 \times 10^{2}$ & 5 & $7 \times 10^{-3}$ \\
\hline B & $1.2 \times 10^{-6}$ & $1008^{2}$ & $4 \times 10^{5}$ & 9 & $\times 10^{-3}$ & $\ldots$ & 10 & $2.6 \times 10^{1}$ & 0.8 & $6 \times 10^{-3}$ \\
\hline $\mathrm{C}$ & $1.2 \times 10^{-5}$ & $1008^{2}$ & $4 \times 10^{5}$ & 9 & $\times 10^{-3}$ & $\ldots$ & 10 & $2.6 \times 10^{0}$ & 0.1 & $4 \times 10^{-3}$ \\
\hline $\mathrm{D}$ & $1.2 \times 10^{-4}$ & $1428^{2}$ & $4 \times 10^{5}$ & 9 & $\times 10^{-3}$ & $\ldots$ & 10 & $2.6 \times 10^{-1}$ & 0.003 & $5 \times 10^{-4}$ \\
\hline $\mathrm{E}$ & $1.2 \times 10^{-3}$ & $1428^{2}$ & $4 \times 10^{5}$ & 9 & $\times 10^{-3}$ & $\ldots$ & 10 & $2.6 \times 10^{-2}$ & 0.0001 & $4 \times 10^{-4}$ \\
\hline $\mathrm{F}$ & $1.2 \times 10^{-3}$ & $1428^{2}$ & $4 \times 10^{5}$ & 9 & $\times 10^{-2}$ & $\ldots$ & 10 & $2.6 \times 10^{-2}$ & 0.006 & $1 \times 10^{-2}$ \\
\hline G & $1.2 \times 10^{-3}$ & $1428^{2}$ & $8 \times 10^{5}$ & 9 & $\times 10^{-2}$ & 0.1 & 10 & $2.6 \times 10^{-2}$ & 0.002 & $1 \times 10^{-2}$ \\
\hline $\mathrm{H}$ & $1.2 \times 10^{-3}$ & $1428^{2}$ & $8 \times 10^{5}$ & 9 & $\times 10^{-2}$ & $\ldots$ & 20 & $2.6 \times 10^{-2}$ & 0.004 & $1 \times 10^{-2}$ \\
\hline I & $1.2 \times 10^{-3}$ & $1428^{2}$ & $1.6 \times 10^{6}$ & 9 & $\times 10^{-2}$ & $\ldots$ & 10 & $2.6 \times 10^{-2}$ & 0.004 & $1 \times 10^{-2}$ \\
\hline
\end{tabular}

and (5) to stabilize the density and velocity fields, respectively, at the grid scale (Lyra et al. 2008; McNally et al. 2012; Lyra et al. 2017).

\subsection{Model Parameters}

We conduct eight simulations in total. For five models-runs A-E-we vary the initial gas surface density $\Sigma_{\mathrm{g}, 0}$ according to the values shown in Figure 1. This range of surfaces densities corresponds with surface densities $10^{4}-10^{8}$ times lower than that of the Minimum Mass Solar Nebula at 100 au (Cameron \& Pine 1973; Weidenschilling 1977). For the $\beta$ Pic disk, Brandeker et al. (2004) assume solar abundances to derive a gas surface density of $3.5 \times 10^{-6} \mathrm{~g} \mathrm{~cm}^{-2}$, based on a scale height of $10 \mathrm{au}$ and a mean molecular mass of $2.5 \mathrm{amu}$. Our models have gas surface densities approximately $0.03-300$ times that value. In run $\mathrm{F}$, we duplicate our model with the highest value of $\Sigma_{\mathrm{g}, 0}$ (run E) but increase the total dust mass by a factor of 10; we achieve this by doubling the number of superparticles and by increasing the mass per superparticle by a factor of five.

In run $\mathrm{G}$, we duplicate run $\mathrm{F}$ but add a large Laplacian viscosity to the right-hand side of Equations (5), corresponding with a Shakura-Sunyaev $\alpha$ viscosity of 0.1 (Shakura \& Sunyaev 1973) at the reference orbital radius (100 au). Lyra \& Kuchner (2013) find that viscosity damps the PeI at high wavenumbers. This viscosity is presumably expected from the magnetorotational instability (MRI; Balbus \& Hawley 1991), the wavelengths of which can be small enough to allow for unstable MRI modes in debris disks (Kral \& Latter 2016). Although the activity of the MRI in optically thin disks remains limitedly understood (Kral \& Latter 2016), we include this additional run to explore the effect of an eddy viscosity when photoelectric heating and radiation pressure are both operative.

In run $\mathrm{H}$, we duplicate run $\mathrm{F}$ but double the width of the birth ring from $\sigma_{\mathrm{BR}}=10$ au to $\sigma_{\mathrm{BR}}=20$ au in order to verify that any dust or gas patterns that emerge in run $\mathrm{F}$ do not require a narrow birth ring to form. In run I, we duplicate run $\mathrm{F}$ but double the number of superparticles as a test of model convergence.

The physical parameters that differentiate our models are summarized in Table 1, including the Stokes number of the smallest particle in each run, denoted as $\min (\mathrm{St})$, which is calculated using Equation (2) for $\Sigma_{\mathrm{g}}=\Sigma_{\mathrm{g}, 0}$. Table 1 also provides the spatially averaged dust-to-gas ratio $\epsilon_{\mathrm{d} / \mathrm{g}}$ for $r<300$ au after 400 orbits, denoted as $\left\langle\epsilon_{\mathrm{d} / \mathrm{g}}\right\rangle$, as well as the characteristic vertical geometric optical depth of any dust overdensities after 400 orbits, denoted as $\tilde{\tau}$.

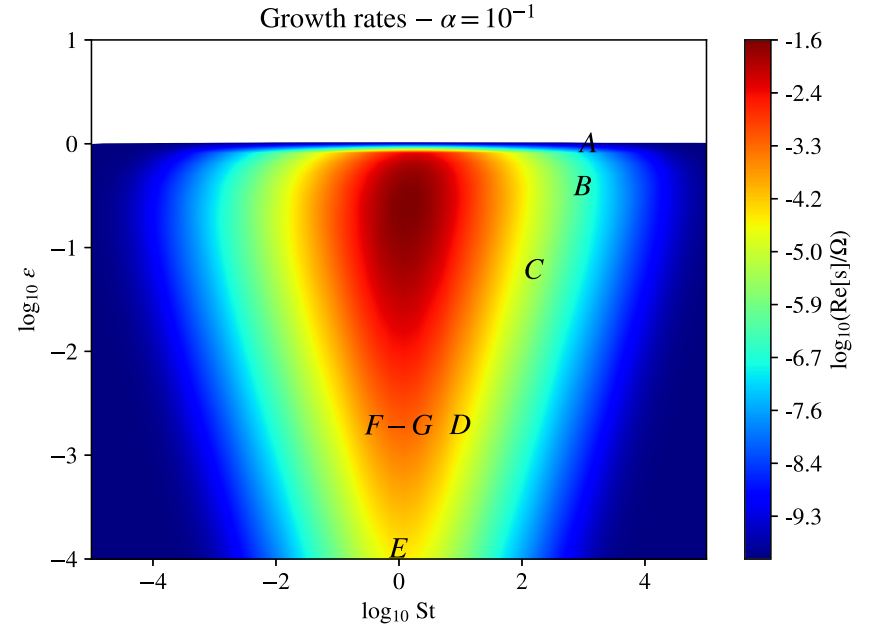

Figure 2. Maximum growth rates of the photoelectric instability as a function of the Stokes number and the dust-to-gas ratio, for a system with turbulent viscosity of $\alpha=0.1$. The runs of this work are labeled in the plot. The Stokes number chosen is in the middle of the range of the actual nonlinear simulations. The actual range of St of each run should span one order of magnitude in each direction. Linear instability for dust-to-gas ratio above unity does not exist.

In each model, the gas is assumed to be initially uniformly distributed throughout the disk between 50 and 800 au from the central star. The gas temperature is also uniform throughout the disk. This yields no global pressure gradient, allowing us to isolate the effects of photoelectric heating; specifically, it allows us to attribute any radial dust drift to the radiation pressure and the PeI. Lyra \& Kuchner (2013) show that for the radiation pressure-free case, the photoelectric instability generates dust rings in the presence of a global pressure gradient and the streaming instability.

We run each model for 400 orbits, which is a sufficient amount of time to determine whether small-to-medium grains can trigger the formation of clumps or other features through the PeI. The largest grains in the lowest-gas runs are so poorly coupled that resolving the PeI growth timescale associated with them would require prohibitively long run times; in these lowgas runs, any participation of large grains in PeI-induced effects would presumably require some complex interplay between small and large grains. For instance, small grains, even unbound ones, could trigger gas overdensities that would yield substantially shorter values of $\mathrm{St}$, leading to better coupling of larger grains. 

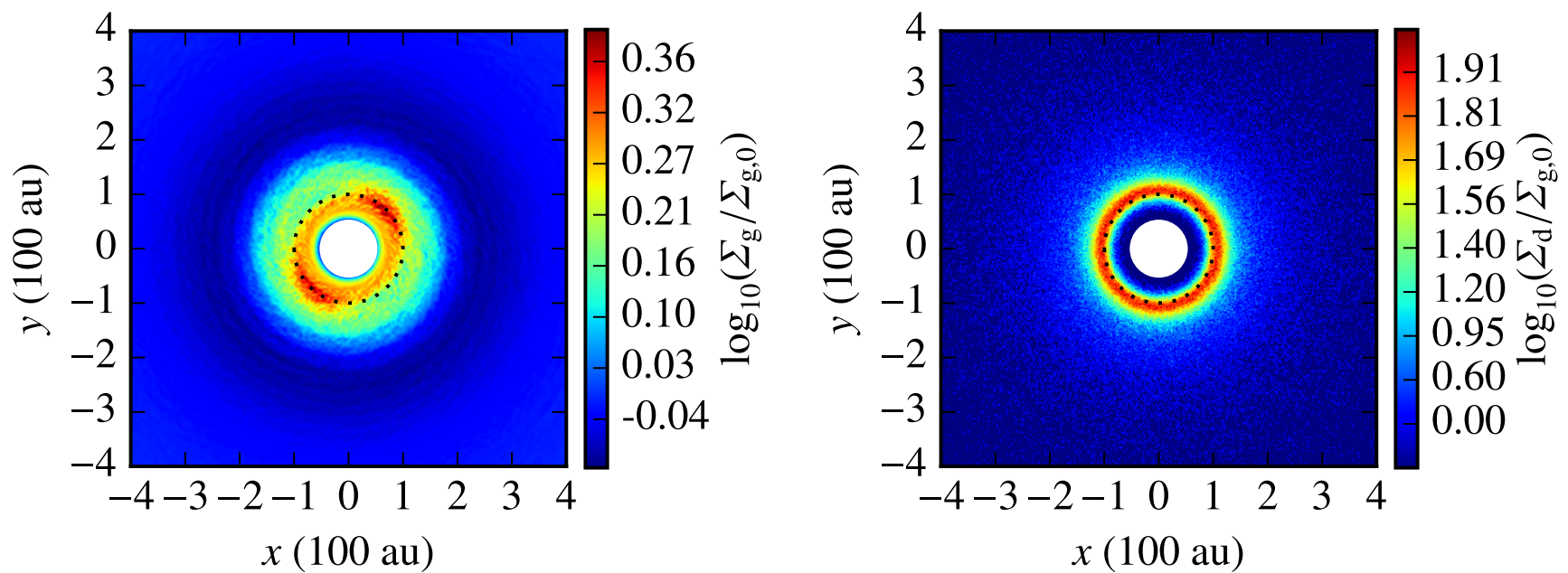

Figure 3. Gas (left panel) and dust (right panel) surface densities after 400 orbits for run A $\left(\Sigma_{\mathrm{g}, 0}=1.2 \times 10^{-7} ; M_{\text {dust }}=9 \times 10^{-3}\right)$. The dashed circle indicates $r_{\mathrm{BR}}$ $(100 \mathrm{au})$. The dust (right panel) shows no indication of perturbation by the gas. The gas vortices (left panel) just outside the birth ring represent a factor of two overdensity, which is still too small to shorten substantially the large values of St for the dust.
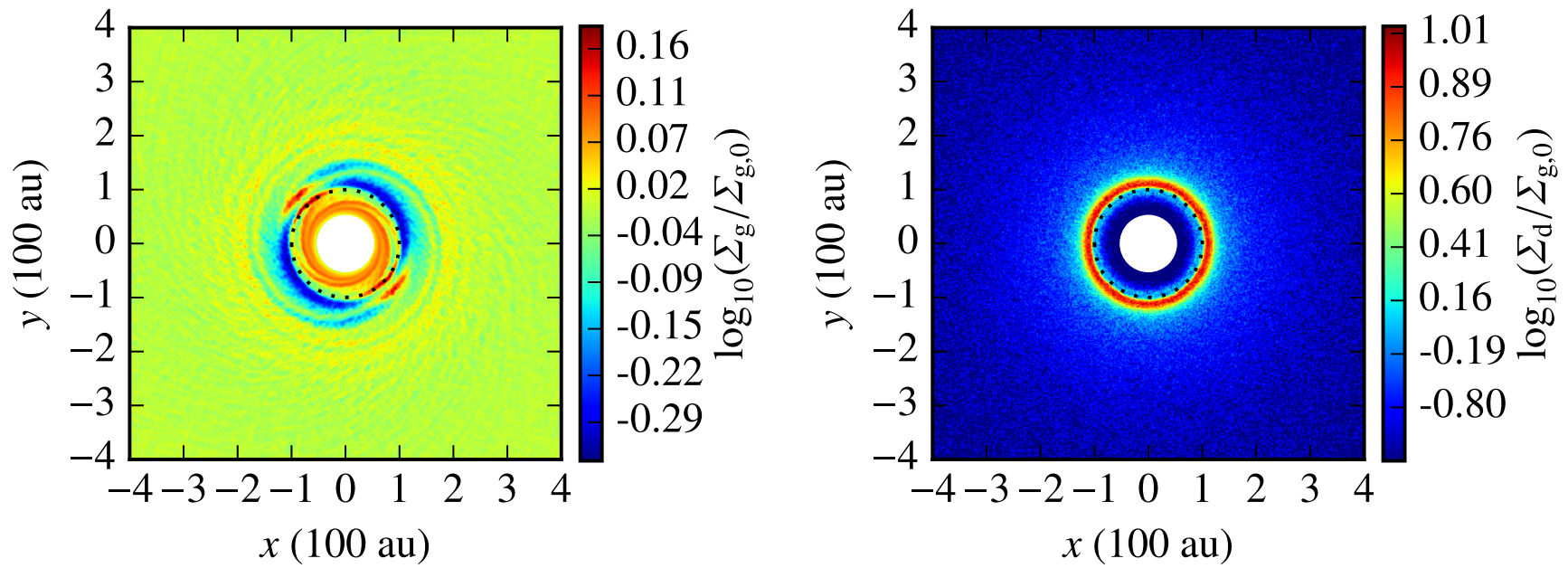

Figure 4. Gas (left panel) and dust (right panel) surface densities after 400 orbits for run B $\left(\Sigma_{\mathrm{g}, 0}=1.2 \times 10^{-6} ; M_{\text {dust }}=9 \times 10^{-3}\right)$. The dashed circle indicates $r_{\mathrm{BR}}$ (100 au). Compared with run A (Figure 3), the dust ring is radially narrower, but still shows no signs of PeI-induced clumping.

\section{Results}

To guide the interpretation of the simulations, we compute the analytical growth rates of the PeI as a function of Stokes number for the range considered. This is done by solving Equations (26)-(29) of Lyra \& Kuchner (2013). Without viscosity, the growth rates would grow unboundedly with the wavenumber, eventually getting unphysically high at the grid scale. In reality, the growth rates drop abruptly when approaching the viscous range. Because of this, we regularize the system with a Laplacian viscosity. The result for $\alpha=0.1$, as in run $G$, is plotted in Figure 2. The figure shows the maximum growth rate $s$ as a function of the Stokes number and the dust-to-gas ratio. The labels $\mathrm{A}-\mathrm{G}$ indicate the minimum Stokes numbers and dust-to gas ratios corresponding to simulations A-G (if runs A-F had Laplacian viscosities rather than hyperviscosities). Above the dust-to-gas ratio unity, no linear instability exists. The symmetry with respect to $\mathrm{St}=1$, as seen in the nonlinear simulations of Lyra \& Kuchner (2013; supplemental Figure 2 of that work) is reproduced. The runs of this work are labeled in Figure 2. The St value chosen as representative of the run is at $r=1$. In each simulation, the range of St should reach an order of magnitude in each direction.

Gas and dust surface densities after 400 orbits at 100 au for runs $\mathrm{A}-\mathrm{H}$ are shown in Figures 3-10. For simplicity, we hereafter use the term "orbits" to indicate orbits at the reference radius $r_{\mathrm{BR}}=100 \mathrm{au}$.

For runs A and B (Figures 3 and 4), we see that a narrow, axisymmetric dust ring forms just outside the birth ring, with a central surface density that corresponds with $\tau \approx 5 \times 10^{-3}$. In the gas, two vortices form just outside the birth ring. In run B, a gas gap appears, along with two additional vortices appear on the opposite side of the gap from the first two, matching them in azimuth. Each vortex orbits at a sub-Keplerian speed, with an orbital frequency approximately $90 \%$ of that expected from the Keplerian rotation.

In order to further investigate this behavior, we plot the gas surface density over time for run B in Figure 11. In the first few dozen orbits, a single vortex $(m=1)$ emerges. In the next several dozen orbits, a gas gap forms, as well as two vortices $(m=2)$ just outside it, positioned $180^{\circ}$ apart from each other in azimuth; the contemporaneity of the formation of the gap and the two inner vortices suggests that the Rossby wave instability 

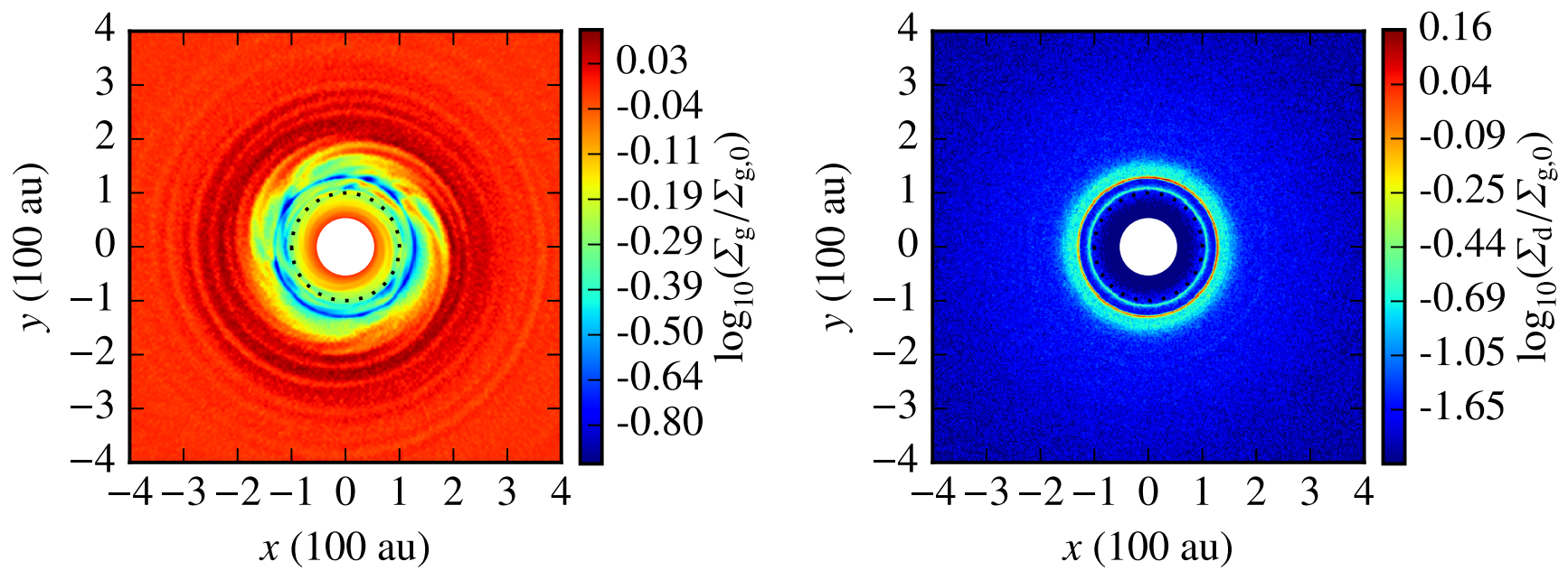

Figure 5. Gas (left panel) and dust (right panel) surface densities after 400 orbits for run $\mathrm{C}\left(\Sigma_{\mathrm{g}, 0}=1.2 \times 10^{-5} ; M_{\text {dust }}=9 \times 10^{-3}\right)$. The dashed circle indicates $r_{\mathrm{BR}}$ (100 au). The dust distribution shows two closely spaced concentric rings, consistent with dust clumping due to the PeI.
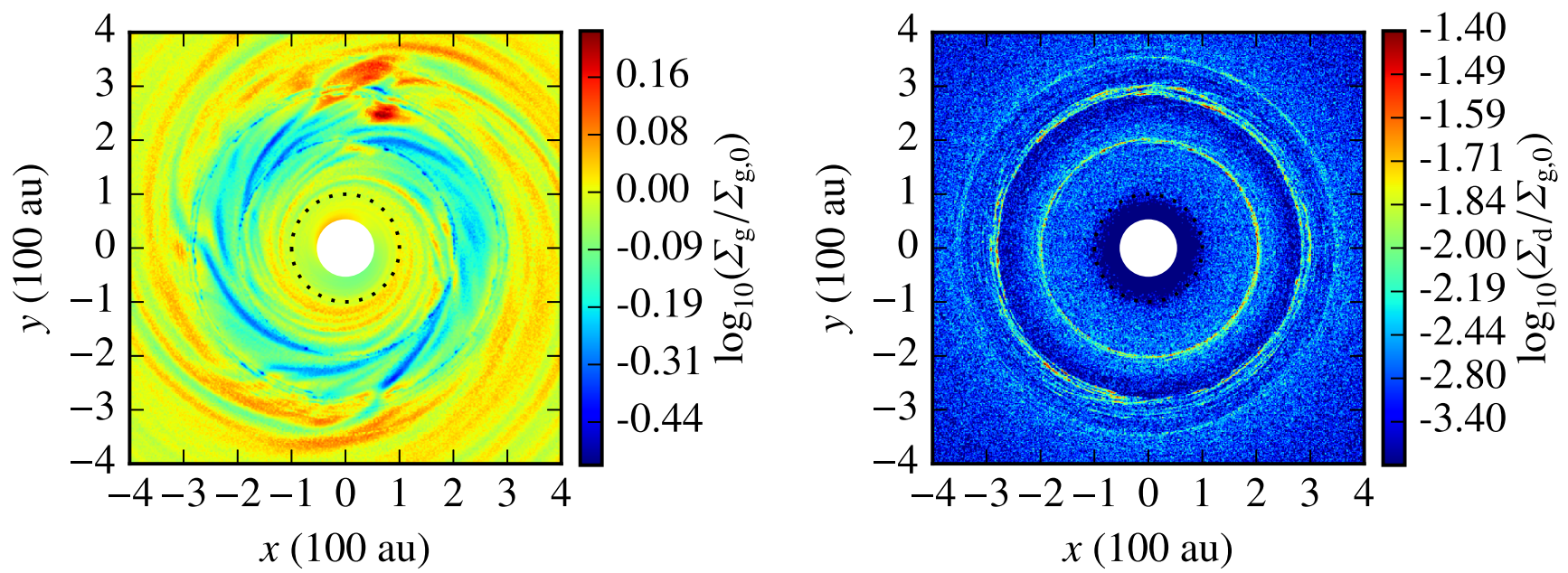

Figure 6. Gas (left panel) and dust (right panel) surface densities after 400 orbits for run $\mathrm{D}\left(\sum_{\mathrm{g}, 0}=1.2 \times 10^{-4} ; M_{\mathrm{dust}}=9 \times 10^{-3}\right)$. The dashed circle indicates $r_{\mathrm{BR}}$ $(100 \mathrm{au})$. The gas distribution (left panel) shows greater nonaxisymmetric structure than in run C (Figure 5). The dust distribution (right panel) shows two prominent ring structures and a third fainter one (the outermost). Ripple structures in the dust spaced a few au apart also appear near each of the three rings, which is strongly reminiscent of the tightly packed arcs and rings seen in the radiation pressure-free models of Lyra \& Kuchner (2013).
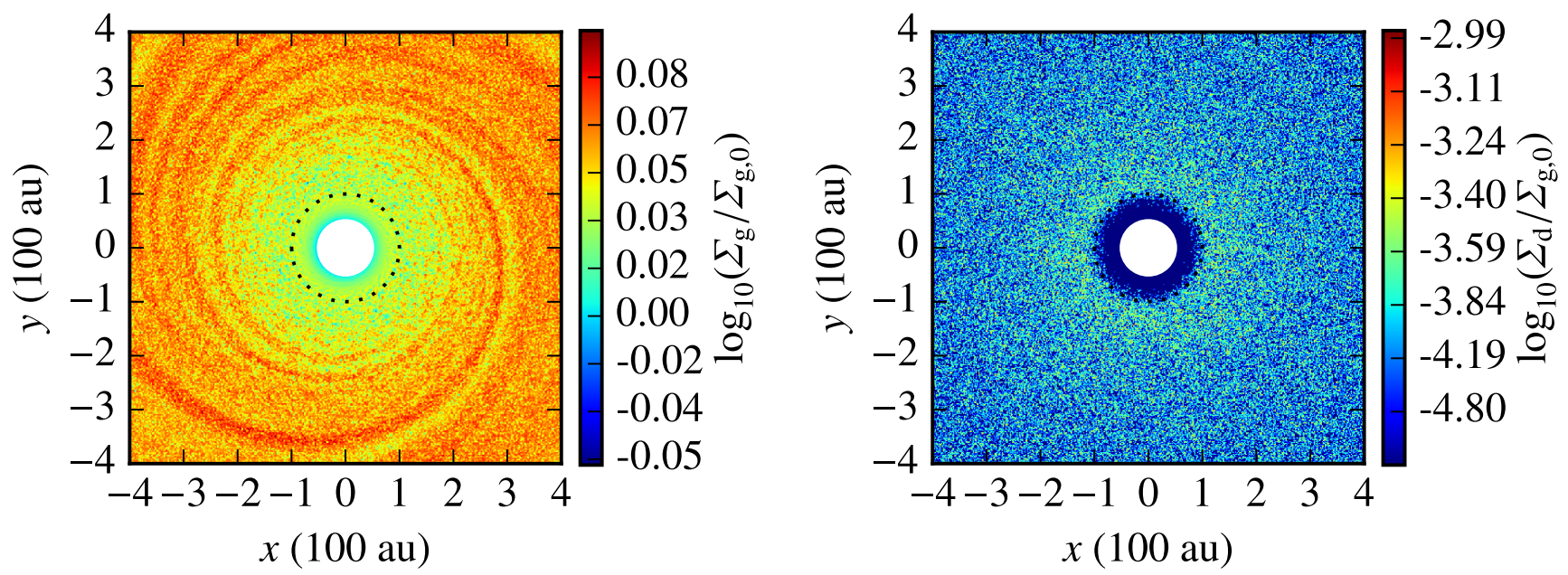

Figure 7. Gas (left panel) and dust (right panel) surface densities after 400 orbits for run $\mathrm{E}\left(\Sigma_{\mathrm{g}, 0}=1.2 \times 10^{-3} ; M_{\mathrm{dust}}=9 \times 10^{-3}\right)$. The dashed circle indicates $r_{\mathrm{BR}}$ (100 au). The lack of structure in the dust distribution (right panel) reflects a long PeI growth time for such a low dust-to-gas ratio. 

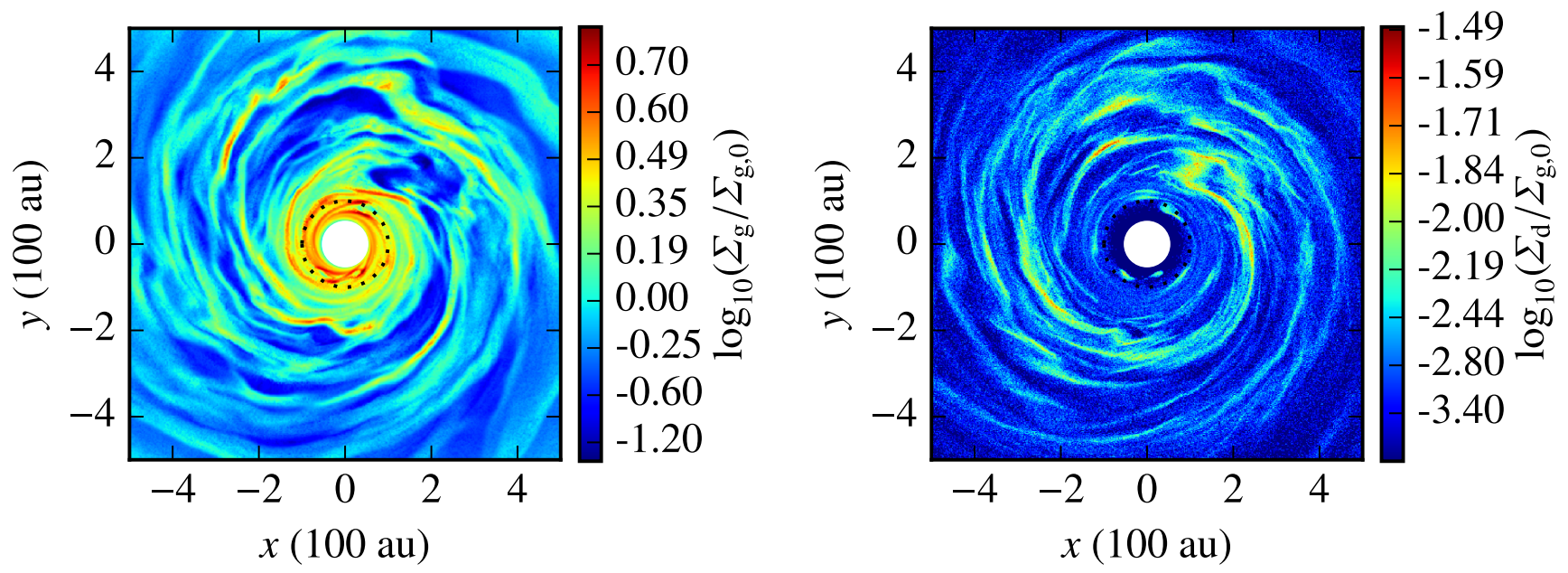

Figure 8. Gas (left panel) and dust (right panel) surface densities after 400 orbits for run $\mathrm{F}\left(\Sigma_{\mathrm{g}, 0}=1.2 \times 10^{-3} ; M_{\mathrm{dust}}=9 \times 10^{-2}\right)$. The dashed circle indicates $r_{\mathrm{BR}}$ (100 au). Increasing the dust mass by a factor of 10 compared with run $\mathrm{E}$ (Figure 7) yields the return of the photoelectric instability. Compared with runs A-E, both the gas and dust (left and right panels, respectively) follow nonaxisymmetric distributions.
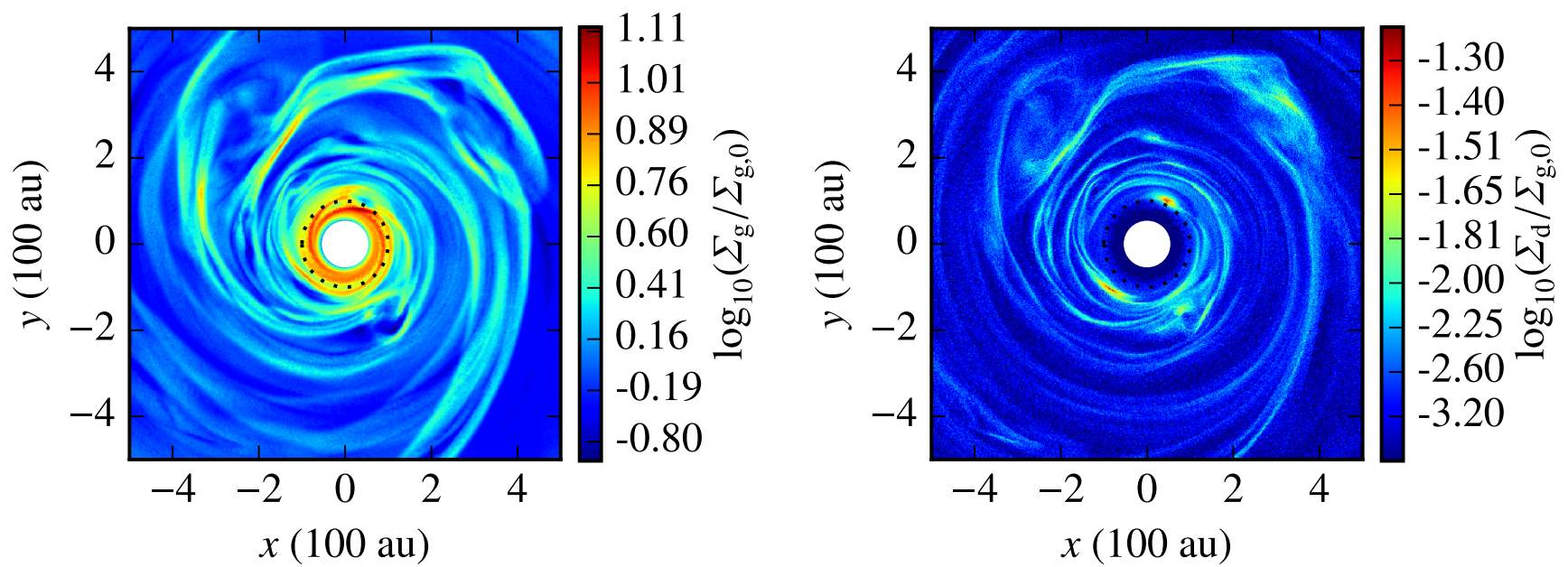

Figure 9. Gas (left panel) and dust (right panel) surface densities after 400 orbits for run $\mathrm{G}\left(\Sigma_{\mathrm{g}, 0}=1.2 \times 10^{-3} ; M_{\text {dust }}=9 \times 10^{-2} ; \alpha=0.1\right)$. The dashed circle indicates $r_{\mathrm{BR}}(100 \mathrm{au})$. The addition of a substantial low-order viscosity does not impede the onset of the photoelectric instability, but it smooths out much of the highfrequency structure seen in run F (Figure 8).
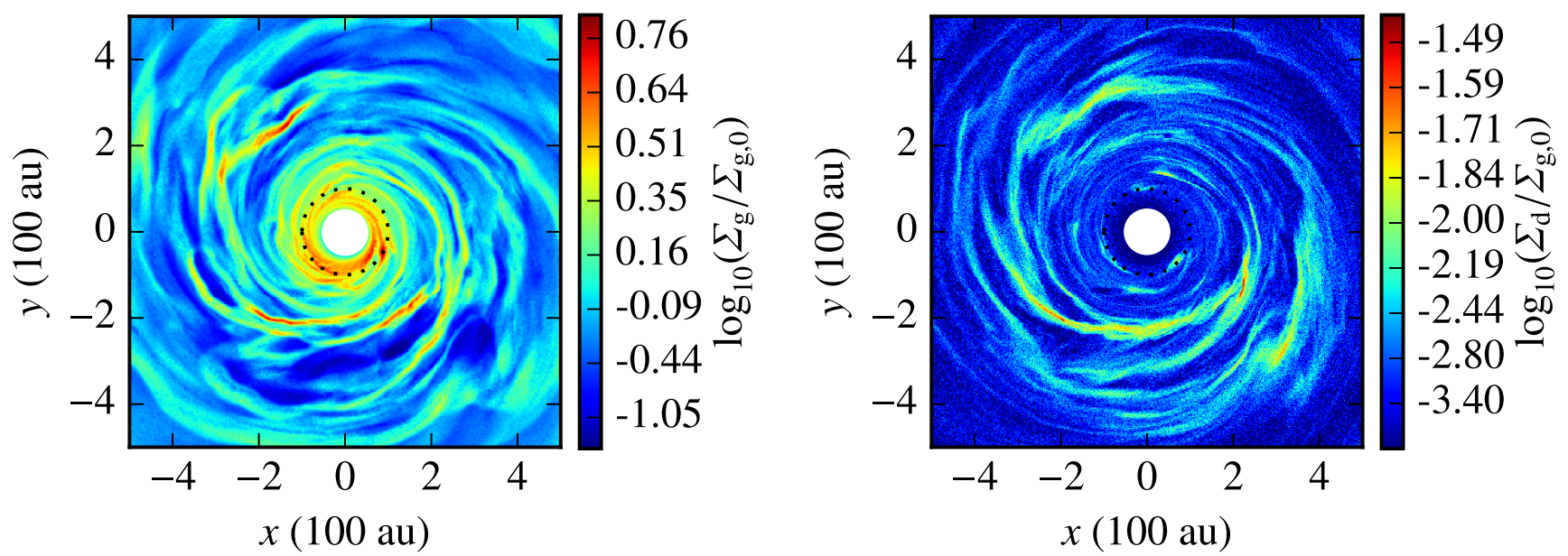

Figure 10. Gas (left panel) and dust (right panel) surface densities after 400 orbits for run $\mathrm{H}\left(\Sigma_{\mathrm{g}, 0}=1.2 \times 10^{-3} ; M_{\mathrm{dust}}=9 \times 10^{-2}\right)$. The dashed circle indicates $r_{\mathrm{BR}}$ $(100 \mathrm{au})$ The conditions for this run are the same as those for run $\mathrm{F}$ (Figure 8), except that the width of the birth ring is doubled from $\sigma_{\mathrm{BR}}=10$ au to $\sigma_{\mathrm{BR}}=20$ au. Dust overdensities, including strong spirals, still form under these conditions, though at slightly (30\%) lower contrast. 


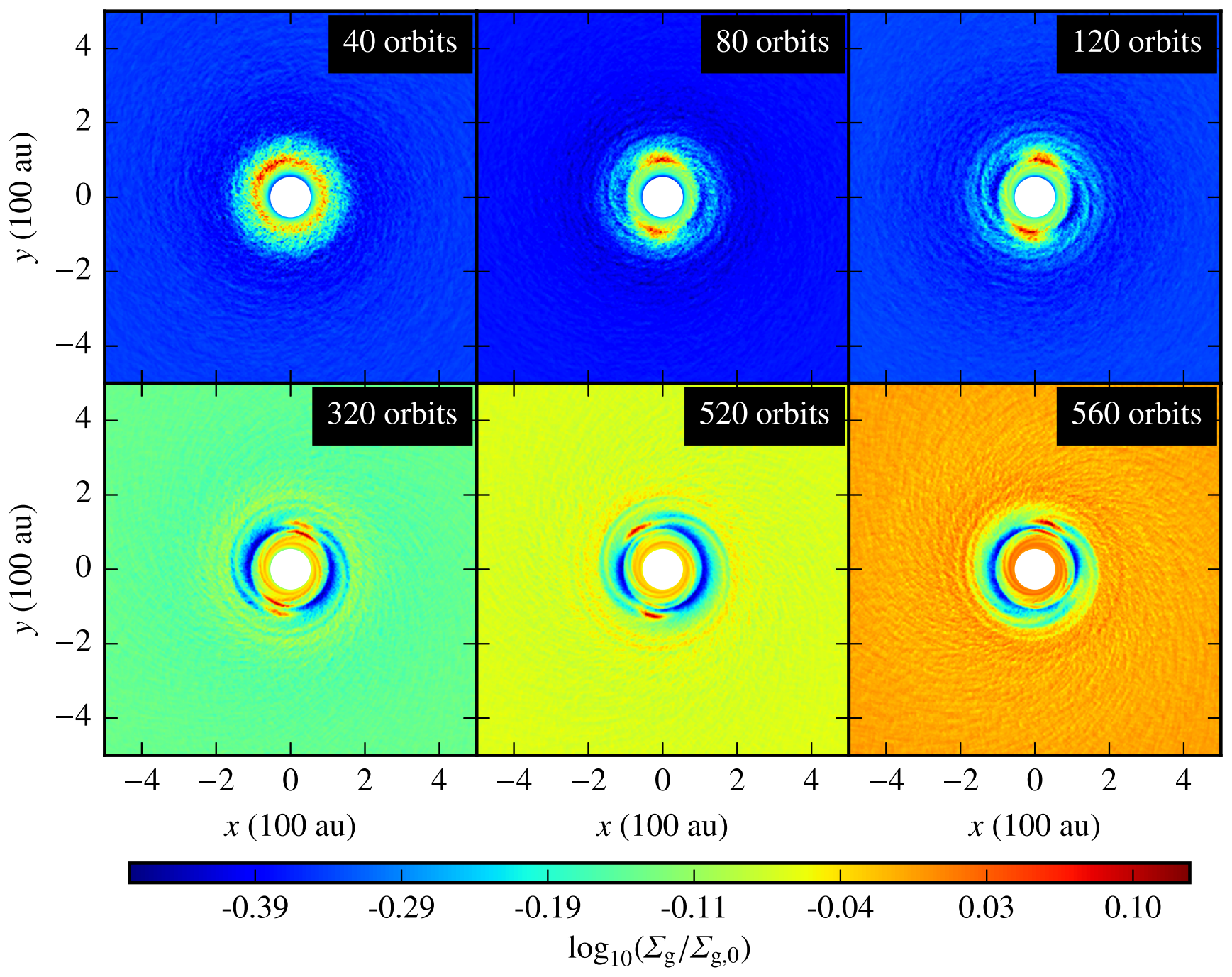

Figure 11. Gas surface density over time for run B. After 560 orbits, only one vortex remains following a long-lived $m=2$ pattern that emerges after dozens of orbits.

(Lovelace et al. 1999) may be responsible. For the next several hundred orbits, two additional "matching" vortices appear just within the orbit of the inner gap edge, keeping pace with the outer vortices. After 520 orbits, the outer vortices have begun to migrate and the inner vortices are much less pronounced, and after 560 orbits only one inner-outer vortex pair remains.

In runs $\mathrm{A}-\mathrm{C}$ (Figures 3-5), we see that the increasing value of $\Sigma_{\mathrm{g}, 0}$ (thus reducing St) results in a narrow gas gap whose depth increases with $\Sigma_{\mathrm{g}, 0}$. In run D (Figure 6), we see a shallower, wider gap threaded by several spiral arm structures (not seen in the models of Lyra \& Kuchner 2013).

In runs $\mathrm{C}$ and $\mathrm{D}$ (Figures 5 and 6), compared with runs $\mathrm{A}$ and $\mathrm{B}$, the gas distributions are less smooth and contain nonaxisymmetric clumps and arcs. The dust distributions show concentric rings with factor of 10-20 dust enhancements, reminiscent of the models of Lyra \& Kuchner (2013), though there a fewer rings in runs $\mathrm{C}$ and $\mathrm{D}$, which have two and three main ring structures, respectively. The central optical depths of these rings are of order $10^{-3}$, with the exception of the outermost ring in run D (Figure 6), which is about an order of magnitude fainter. In run C, the anticorrelation of gas and dust just outside the birth ring is reminiscent of the dust-gas anticorrelation seen in the models of Lyra \& Kuchner (2013).
In run $\mathrm{D}$, the dust rings are accompanied by more highfrequency structures, including some localized ripple patterns (a few au in scale) that are strongly reminiscent of the tightly packed arcs and rings seen in the Lyra \& Kuchner (2013) models. These rings are long-lived, having begun to form after only a few tens of orbits. Though the gas responds to the dust on dynamical timescales due to photoelectric heating, in runs $\mathrm{A}-\mathrm{D}$, the Stokes numbers are high enough that the bound grains $\left(\beta_{\mathrm{PR}}<1 / 2\right)$ respond to the gas only on much longer timescales; grains on low-eccentricity orbits see the azimuthally averaged gas distribution over the course of many orbits, not the gas's spiral structure. This asymmetrical coupling promotes the formation of dust rings. But note that some of the fine structure in the rings, like the bifurcation at the one o'clock position, seems to correspond with clumps in the gas.

No indications of the photoelectric instability emerge after 400 orbits for run E (Figure 7). As shown in Figure 2, the growth rate for run $\mathrm{E}$ is centered at the maximum value of $s \approx 10^{-5} \Omega$. After 400 orbits, this low growth rate amounts to a mere $2 \%$ amplification. Runs D and C (though centered at the same low level of growth rate) and B (centered at even lower) reach to the left of the diagram and into regions of higher growth rates, as much as $10^{-2} \Omega$, (million-fold amplification in $\approx 220$ orbits). Run A, though also centered at a low value of 
growth rate, and does not reach too deep into regions of high growth rate, may be nonlinearly amplified due to the high dustto-gas ratio.

For run $\mathrm{F}$ (Figure 8), we find that increasing the total dust mass compared with run $\mathrm{E}$ yields the return of the photoelectric instability. The photoelectric instability radically transforms both the gas and dust distributions; the dust clumps and arcs seen in the right panel of Figure 8 correspond with $\tau \approx 10^{-2}$, representing a factor of 5-10 enhancement over the local dust surface density.

Qualitatively, the behavior of run $\mathrm{F}$ is quite different from that of run $\mathrm{D}$, where the dust remains in coherent rings. As seen in Figure 1, for run D, bound dust grains are weakly coupled with the gas, generating the rings discussed above. In run $\mathrm{F}$, a large range of grain sizes is both bound and well-coupled to the gas. By setting $\mathrm{St}=1$ and $\beta=1 / 2$ in Equation (3), we find that the threshold for having grains that are both bound and well-coupled occurs at a gas surface density of

$$
\Sigma_{\mathrm{g}}>1.8 \times 10^{-4} \mathrm{~g} \mathrm{~cm}^{-2}\left(\frac{L_{\odot}}{L_{\star}}\right)\left(\frac{M_{\star}}{M_{\odot}}\right) .
$$

One possible hypothesis to explain the qualitatively different results between runs $\mathrm{D}$ and $\mathrm{F}$ is that grains that are both bound and well-coupled generate spiral patterns, while grains that are not well-coupled to the gas (i.e., on timescales that are short compared to an orbital period) form ring-like patterns. If this interpretation should hold, we might expect the gas surface density threshold in Equation (11) to represent a transition between two different families of disk patterns. Another possible explanation is that the higher level of dust in run $\mathrm{F}$ creates a greater drag backforce against the gas, destabilizing it, leading to results resembling those of the backreaction-free model of Lyra \& Kuchner (2013).

The gas and dust distributions for run F (Figure 8) are strikingly similar to the results of the Lyra \& Kuchner (2013) model that excludes drag backreaction on the gas. In the absence of radiation pressure, where both the gas and dust move on roughly circular orbits, the expansion of gas due to high pressures induced by photoelectric heating will undergo a Coriolis rotation. In models with backreaction, this rotation is opposed by the backreaction from the dust, and axisymmetry of the system is maintained. When a dust grain is placed on a highly eccentric orbit by radiation pressure, it may create a local pressure maximum but not linger in a nearby circular orbit where it can stabilize the gas through drag backreaction. This may provide a second possible explanation for the difference between runs $\mathrm{D}$ and $\mathrm{F}$ discussed above, especially given the higher mass of dust used in run $\mathrm{F}$, which may lead to drag backforces that are strong enough to destabilize gas from a circular orbit.

In Figure 12, we show the dust surface density every 50 orbits for 400 orbits. We find that the photoelectric instability sets in quickly, but has no obvious secular effect on the global structure of the disk. The behavior is dominated by transient clumps and arcs that appear and disappear on timescales of orbits to dozens of orbits.

In order to confirm that the dust structures seen in run $\mathrm{F}$ (Figure 8) are conclusively attributable to the photoelectric instability, we run two additional models: one without photoelectric heating, and one with an increased dust resolution to ensure model convergence (run I). For run I, we double the number of dust superparticles while reducing the mass of each superparticle by half in order to maintain the same overall dust mass. The run without photoelectric heating shows no clumping behavior, and instead yields a smooth, structureless dust distribution, as one would expect. Run I, on the other hand, shows the same clumping behavior as seen in run F, suggesting that the resolution we use in runs $\mathrm{A}-\mathrm{F}$ is sufficient. Gas and dust surface densities after 400 orbits are shown in Figure 13.

In run $\mathrm{H}$, we duplicate run $\mathrm{F}$ (Figure 8 ) but double the birth ring width to verify that the dust clumping behavior of run $\mathrm{F}$ does not require a narrow birth ring. Dust and gas surface densities after 400 orbits for run $\mathrm{H}$ are shown in Figure 10. We find that the enhancement in the dust-to-gas ratio produced by the photoelectric instability is $\sim 30 \%$ weaker in run $\mathrm{H}$ compared with run F (see Table 1), but the qualitative results - nonaxisymmetric, PeI-induced dust concentrations-are the same.

Lyra \& Kuchner (2013) find that in the presence of drag with backreaction and photoelectric heating, the gas and dust mutually displace each other, leading to alternating rings of gas and dust throughout the disk. It is apparent in Figures 3-5 that when a small amount of gas is present, dust displaces it, creating a gas gap. In order to test whether gas and dust anticorrelate when larger quantities of gas are present, we plot the product of and (specifically, their mean-subtracted and standard deviation-normalized values) for run $\mathrm{F}$ after 400 orbits in Figure 14. We find that the dust and gas correlate (red regions) and anticorrelate (blue regions), in roughly equal measure.

We find that the inclusion of a very strong low-order gas viscosity term in run $G$ (Figure 9) yields results that are fairly similar to the no-viscosity case (run F; Figure 8). It does, however, lead to the smoothing of some of the small-scale structure in both the gas and dust distributions seen in run F; dust surface density enhancements are of the order factor of 5 , which is somewhat smaller than seen in run F. The small, transient dust clumps seen in run $\mathrm{F}$ are less numerous and less elongated in run $\mathrm{G}$, but are nonetheless present and also appear and disappear over orbital timescales. This result is consistent with the predictions and models of Lyra \& Kuchner (2013), where viscosity suppresses the PeI at high wavenumbers, smoothing small-scale structure but not impeding the ability of the PeI to reshape substantially the disk. This difference between the results of runs $F$ and $G$ suggests that resolved images of debris disks may be able to provide constraints on the turbulent behavior (i.e., the effective viscosity) of debris disk gas.

In order to explore the roles played by different grain sizes in creating and sustaining the structures seen in the right-hand panels of Figures 3-9, we examine the radial distributions of dust in several bins of grain size, choosing runs $\mathrm{A}$ and $\mathrm{F}$ as representative cases. The top, middle, and bottom panels of Figure 15 show radial dust distributions for run A after 400 orbits, run $\mathrm{F}$ after 20 orbits, and run $\mathrm{F}$ after 400 orbits, respectively.

The radial dust distributions shown in the top panel of Figure 15 confirm that it is large particles (i.e., small $\beta$, corresponding with nearly circular orbits) that constitute the dust ring seen in run A (Figure 3). This is consistent with the fact that these sharp rings take dozens of orbits to form, reflecting the large values of St for large grains. The small 

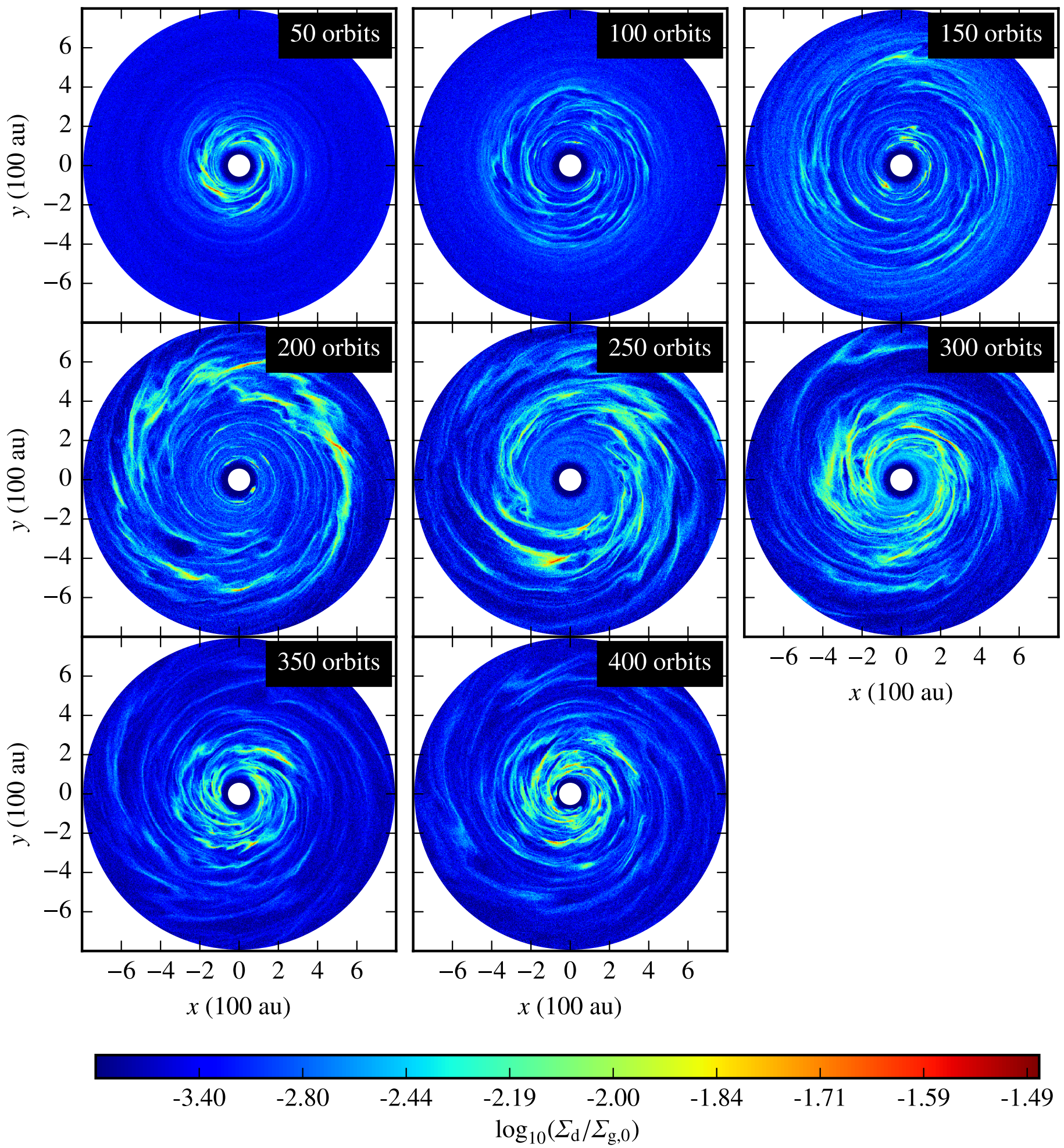

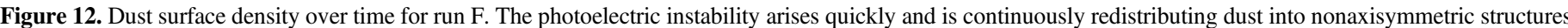

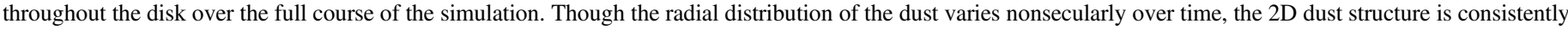
dominated by clumps and arcs that form and dissipate on short timescales of $\approx 1-10$ orbits.

values of $\beta$ place these large grains on a circular orbit, keeping them close to the birth ring.

In the middle and bottom panels of Figure 15, we identify two important grain size-dependent effects. The first, seen in the bottom panel, is that even large grains $(7-10 \mu \mathrm{m})$ migrate outward from their low-eccentricity orbits in and around the birth ring, having now had enough time $(t>\mathrm{St} \cdot \Omega \mathrm{K})$ to be entrained in PeI-induced flows. The second is that smaller grains are more efficiently entrained by the denser gas, leading ordinarily unbound particles $(\beta<1 / 2)$ to remain in the disk. The middle panel shows the grain distribution for run $\mathrm{F}$ after only 20 orbits, at which point the photoelectric instability is just forming. Small-to-medium grains $(0.1-3 \mu \mathrm{m})$, as they are being blown outward by radiation pressure, accumulate outside the 

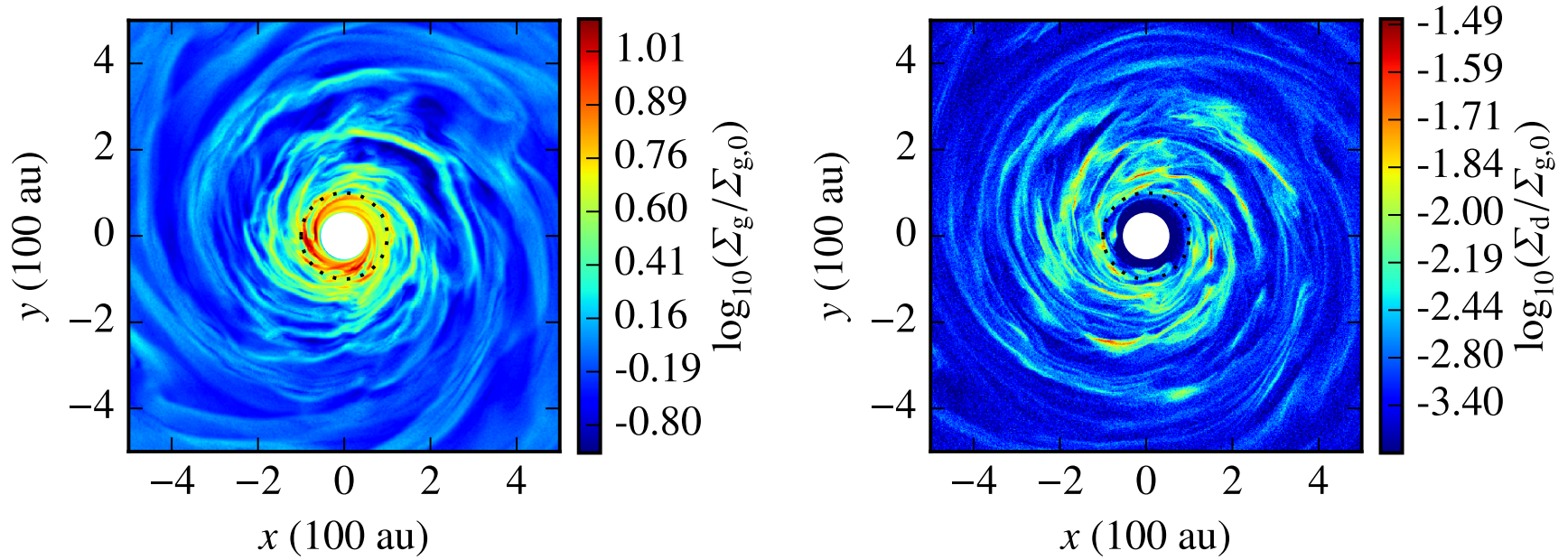

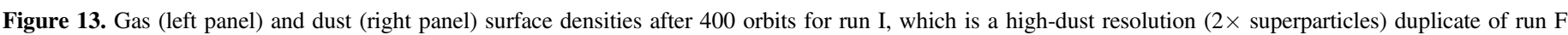

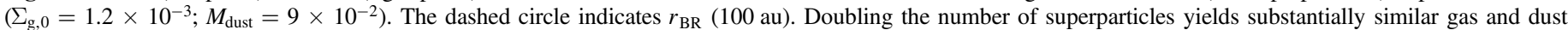

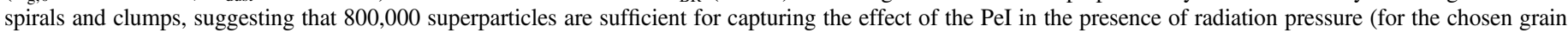
size distribution).

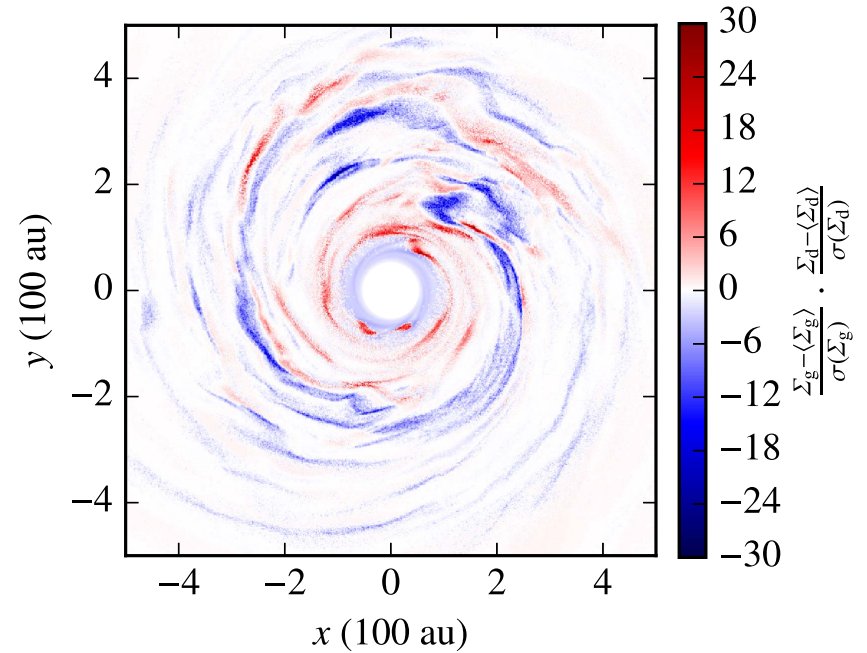

Figure 14. Gas-dust correlation map for run $\mathrm{F}\left(\Sigma_{\mathrm{g}, 0}=1.2 \times 10^{-3} ; M_{\mathrm{dust}}=\right.$ $9 \times 10^{-2}$ ). The gas and dust correlate (red regions) and anticorrelate (blue regions) in equal measure, contrary to the alternating (i.e., anticorrelating) patterns of gas and dust seen in the models of Lyra \& Kuchner (2013).

birth ring and trigger the photoelectric instability. Meanwhile, larger and therefore more weakly coupled grains migrate outward by the gas drag over the course of orbits to tens of orbits. The dominance of large grains seen in the bottom panel of Figure 15 suggests that while small, unbound grains help to trigger the photoelectric instability and participate in the resulting transient structures, many of them will ultimately be blown out, and the dust structure seen in Figure 8 eventually becomes dominated by larger, bound grains. This suggests that even in disks around more massive (say, A-type) stars, the extreme radiation pressure on dust grains (and the subsequent lack of bound grains) does not necessarily inhibit the formation of the photoelectric instability.

\section{Limitations}

The models presented in this work are the first models of optically thin disks to simultaneously include aerodynamic dust-gas drags with backreaction, dust-gas photoelectric heating, and stellar radiation pressure on dust. Nonetheless, there are many physical processes not included in our models that should be explored in future work, as well as a number of processes included in the current work that merit further study.

\subsection{MRI}

The MRI may operate efficiently in debris disks (Kral \& Latter 2016). The inclusion of a low-order viscous term in run $\mathrm{G}$ produces an observably different dust distribution from run $\mathrm{F}$; the role of MRI-induced turbulence in redistributing gas, and subsequently dust, should be explored in detail. The 3D models needed to explore fully the MRI in debris disks will also help to explore the effect of mutually differing gas and dust scale heights.

\subsection{Gas-Gas versus Dust-Gas Photoelectric Heating}

Future studies should explore the competing roles of gas-gas and dust-gas photoelectric heating to determine the precise realm of the disk parameter space in which dust-gas photoelectric heating is dynamically important. Kral et al. (2016) point out that in a carbon-rich disk like that around $\beta$ Pic, heating by photoelectrons released from carbon may overwhelm the heating produced by those released from dust. This in turn may inhibit the photoelectric instability by inhibiting the formation of pressure maxima.

\subsection{Gas Species}

In the current work, we model only a single gas species. However, as noted by Xie et al. (2013), different gas species can experience different values of $\beta$. The presence of a modest radiation force on a certain gas species with, say, $\beta=0.2$ would significantly affect the mutual velocity of that species with the dust, potentially altering the effects of the PeI.

\subsection{Poynting-Robertson Drag}

Future investigations should explore the role of the Poynting-Robertson drag in the context of the photoelectric instability. The findings of Lyra \& Kuchner (2013) suggest that even large, poorly coupled grains will eventually give rise to 

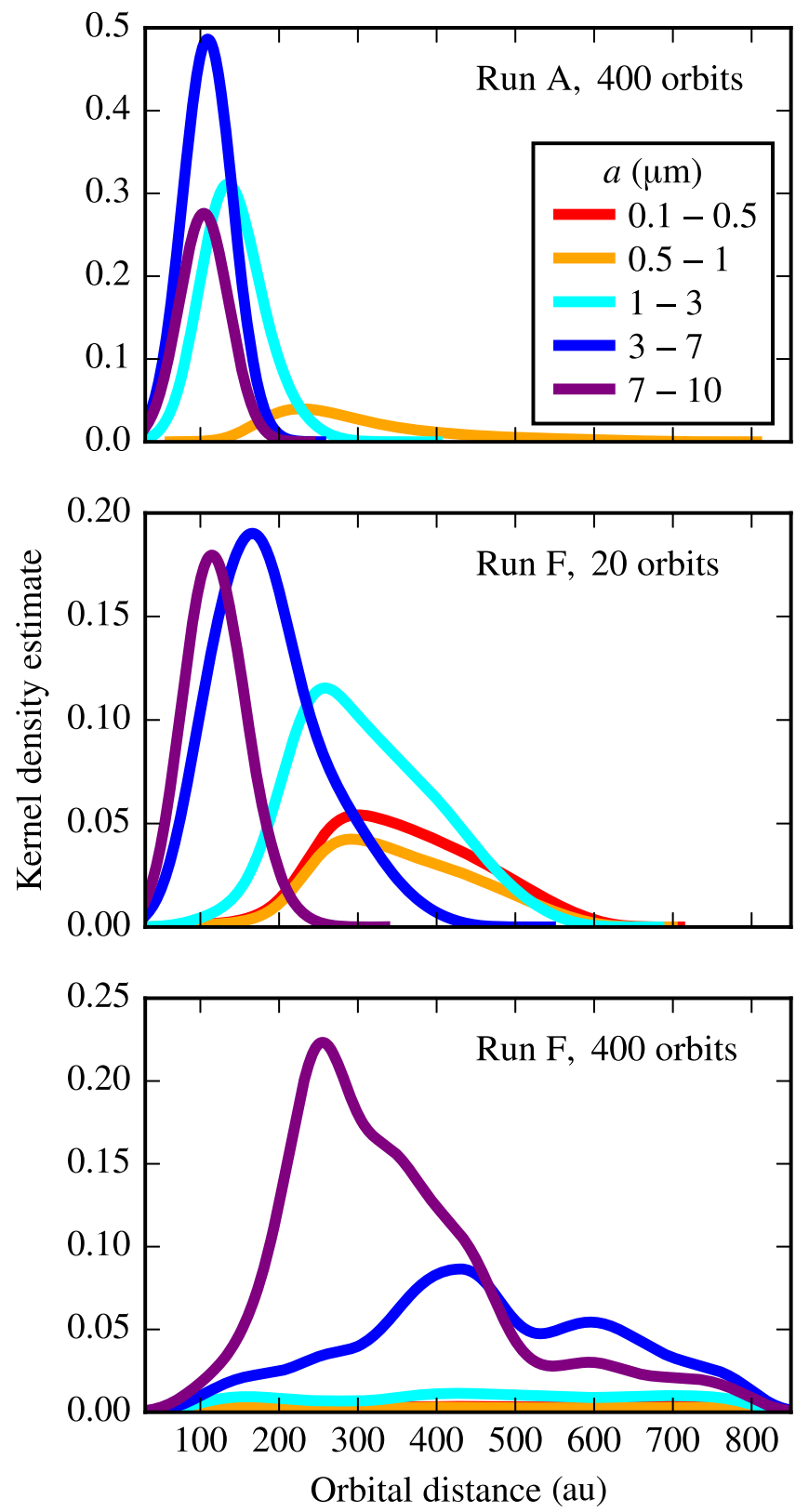

Figure 15. Radial distributions for 5 grain size bins for run A after 400 orbits (top panel), run $\mathrm{F}$ after 20 orbits (middle panel), and run $\mathrm{F}$ after 400 orbits (bottom panel). When little gas is present (run A, top panel), large and bound grains remain on circular orbits near the birth ring and are almost completed unperturbed by the sparse gas. At higher gas densities (run F), small grains trigger the photoelectric instability outside the birth ring (middle panel), but the resulting gas flows also entrain larger grains, carrying them away from the birth ring and eventually dominating nearly the full radial extent of the disk (bottom panel).

the photoelectric instability, but on very long timescales, an inward drift due to the Poynting-Robertson drag could inhibit or affect this process.

\subsection{Stellar Spectral Type}

In the current work, we have only modeled disks around solar-type stars. Around A-type stars, where many debris disks (including those with unusual morphologies) are found, the radiation force on dust grains will be considerably greater. This means that the bound grains will be larger (though the results presented in Figure 15 suggest that even unbound grainssmall and well-coupled-can trigger the PeI on their way out of the disk). Large and, therefore weakly coupled grains can generate and participate in the photoelectric instability; however, the resulting structures will take longer to emerge (a likely explanation for the lack of dust clumping in runs $\mathrm{A}$ and B). It is possible, for instance, that the spatial and temporal frequencies of any nonaxisymmetric structures resulting from gas-dust interactions involving large grains will be larger due to the larger values of St.

\subsection{Grain Properties}

We have assumed smooth, spherical grains in this work. Future works should relax this assumption and explore a range of grain shapes, compositions, etc., which will affect both $\beta_{\mathrm{PR}}$ and St for each grain.

\subsection{Photophoresis}

Grains embedded in gas can be accelerated by photophoresis, which is the force associated with the asymmetric gas temperature distribution surrounding an illuminated particle (e.g., Krauss \& Wurm 2005; McNally \& Hubbard 2015). For the gas densities and grain sizes assumed in the models in this work, the photophoretic force appears to be $<1 \%$ of the radiation pressure force, based on Figure 1 in Krauss \& Wurm (2005). Models exploring disks with higher densities and larger grains, perhaps at smaller orbital distances, should model photophoresis.

\section{Conclusions}

We have produced hydrodynamical models of optically thin disks with gas and dust, while simultaneously incorporating photoelectric heating and stellar radiation pressure on dust grains. We find the following:

1. The emergence of the photoelectric instability (Klahr \& Lin 2005; Besla \& Wu 2007; Lyra \& Kuchner 2013) is not impeded by the radiation pressure associated with a solar-type star.

2. The PeI growth rate is small for gas surface densities $\Sigma_{\mathrm{g}}<10^{-6} \mathrm{~g} \mathrm{~cm}^{-2}$, but fast enough at higher gas surface densities that we see the PeI create dust density enhancements of up to a factor of 20 in our runs of just 400 orbits.

3. For a modest level of gas $\left(10^{-6} \mathrm{~g} \mathrm{~cm}^{-2}<\Sigma_{\mathrm{g}}<\right.$ $10^{-5} \mathrm{~g} \mathrm{~cm}^{-2}$; runs $\mathrm{C}$ and D), the photoelectric instability gives rise to axisymmetric dust rings over the course of dozens of orbits, as well as the azimuthal structure in the gas. The dust and gas show the anticorrelation behavior predicted by Lyra \& Kuchner (2013).

4. For a higher level of gas $\left(\Sigma_{\mathrm{g}}=10^{-4} \mathrm{~g} \mathrm{~cm}^{-2}\right.$; run E) and a similar dust-to-gas ratio $\epsilon_{\mathrm{d} / \mathrm{g}}$ (runs $\mathrm{F}$ and $\mathrm{G}$ ), the PeI emerges over the course of dozens of orbits, leading to an erratic spiral structure throughout the disk (resembling the backreaction-free model of Lyra \& Kuchner 2013), with a small-scale structure appearing and disappearing over the course of orbits. In these models, there is no overall tendency of the dust and gas to anticorrelate.

The value of $\Sigma_{\mathrm{g}}$ required to generate the PeI on short timescales in a given system, however, will depend on many parameters, including the spectral type, total dust mass, initial 
gas profile, and grain size range. In some of our models with a higher level of gas $\left(\Sigma_{\mathrm{g}}=10^{-4} \mathrm{~g} \mathrm{~cm}^{-2}\right.$; run $\left.\mathrm{E}\right)$, but a low effective dust-to-gas ratio $\epsilon_{\mathrm{d} / \mathrm{g}}$, we found the PeI growth rate to be too slow to be captured by our simulations.

Previous models of debris disks with gas have lacked the physics to capture hydrodynamical instabilities like the PeI. Thébault \& Augereau (2005) and Krivov et al. (2009) present models of debris disks with gas spanning a range of gas surface densities similar to the range used in the current work (though other key parameters vary between these works, such as stellar spectral type and the initial radial profile of the gas). In both cases, dust grains generated in a birth ring experience aerodynamic drag with a static gas cloud, precluding the emergence of the PeI, which requires dust-gas heating and a drag backreaction. These 1D models yield dust density distributions that decrease smoothly and monotonically with orbital radius, making them readily distinguishable from the clumps, arcs, and narrow rings seen in the models presented in Section 4.

Other models of optically thin disks reveal more complex morphologies that are not as readily distinguished from the results presented in this work. For instance, the models of cataclysmic massive body collisions produced by Kral et al. (2015) produce nonaxisymmetric structures that could be difficult to distinguish from the dust distributions shown in Figure 8 and 9, especially for disks in an edge-on viewing configuration. Nonetheless, the models of Kral et al. (2015) show a smoother, more organized structure compared with the higher-frequency, more erratic structure seen in runs $F$ and $G$ (Figures 8 and 9) and also do not show concentric, axisymmetric rings, as in runs $\mathrm{C}$ and $\mathrm{D}$. Comparisons of dust distributions at multiple wavelengths for a given disk could also help to disentangle these two effects, given that differential behavior by grain size should be greater for aerodynamic effects than the gravitational ones.

Augereau \& Papaloizou (2004) model a circumstellar debrisonly disk with an external stellar perturber in order to study the origins of the spiral morphology of the HD 141569 disk. In general, their models produce a smooth spiral structure in the circumprimary disk; however for perturbers with eccentric orbits, the structure produced in the disk is less smooth and is somewhat reminiscent of the dust distributions seen in runs $\mathrm{F}$ and $G$ (Figures 8 and 9). Here again, multiwavelength image comparisons may be necessary to distinguish these models. Given that none of the numerical models so far produced of the PeI have given rise to smooth spiral arms of dust (Lyra \& Kuchner 2013 and the current work), it would seem that perturbation by a massive companion is currently a more plausible explanation for such structures (though this may change as the PeI is modeled throughout a larger parameter space).

For a number of other observed disk morphologies, however, the PeI may provide a more plausible explanation than the presence of a massive perturber. The resemblance of the dust distributions for runs $\mathrm{C}$ and $\mathrm{D}$ (Figures 5 and 6) to the concentric rings seen in the scattered light profile of the disk around HIP 73145 (spectral type A; Feldt et al. 2017) is particularly striking. The large grains are relatively compactly distributed, while small grains experience considerable radiation pressure. Though we do not model A-type stars in the current work, our models suggest that the photoelectric instability provides a promising explanation for such features, which can be triggered by unbound grains.

Also, the nonaxisymmetric clumps and spiral arms seen in the gas distributions in several of our models suggest that interactions between gas and small-to-medium grains could underlie the asymmetric structures seen in the 49 Ceti and AU Mic edge-on disks (Boccaletti et al. 2015; Hughes et al. 2017), though explanations for the AU Mic moving blobs involving only rocky body collisions (no gas) have also been proposed (Chiang \& Fung 2017; Sezestre et al. 2017). We also underscore that, so far, the PeI is the best candidate for producing arcs, which is a feature that is not predicted from a planet-disk interaction. Lyra \& Kuchner (2013) predicted that the PeI leads to arcs before the discovery of these features in the disk around HD 141569A (Perrot et al. 2016).

The broad resemblance of our models to several observed systems notwithstanding (models that include more physical details-magnetic fields, multiple gas species, and so on-and that explore a wider range of parameters (especially stellar spectral type)) will help to confirm that the photoelectric instability can indeed provide a plausible explanation for these diverse and intriguing disk morphologies.

Once the interactions of dust, gas, and radiation in optically thin disks are better understood, the signposts of embedded planets can be more accurately modeled. The substantial effect of drag backreaction and photoelectric heating seen in the planet-free models of the current work suggests that planetdisk interactions in optically thin disks may manifest themselves very differently than they do in existing, simpler models. The formation of gaps, rings, and other disk morphologies associated with planets may be inhibited, enhanced, or otherwise affected by the presence of gas.

We thank Yanqin Wu and Ruobing Dong for commenting on an early version of this manuscript. W.L. acknowledges support of Space Telescope Science Institute through grant HST Cycle 24 AR-14572 and the NASA Exoplanet Research Program through grant 16-XRP16 2-0065. M.K. acknowledges support provided by NASA through a grant from the Space Telescope Science Institute (HST Cycle 21 AR-13257.01), which is operated by the Association of Universities for Research in Astronomy, Inc., under NASA contract NAS 5-26555 and support provided by NASA via the NASA Astrobiology Institute Cycle 7 Cooperative Agreement Notice (NNH13ZDA017C) through the Goddard Center for Astrobiology "Origin and Evolution of Organics and Water in Planetary Systems" (Proposal Number 13-13NAI7_2-0032).

\section{ORCID iDs}

Alexander J. W. Richert (iD https://orcid.org/0000-00029613-6863

Wladimir Lyra (iD https://orcid.org/0000-0002-3768-7542

Marc J. Kuchner (i) https://orcid.org/0000-0002-9622-9605

\section{References}

ALMA Partnership, Brogan, C. L., Pérez, L. M., et al. 2015, ApJL, 808, L3 Augereau, J. C., \& Papaloizou, J. C. B. 2004, A\&A, 414, 1153

Balbus, S. A., \& Hawley, J. F. 1991, ApJ, 376, 214

Besla, G., \& Wu, Y. 2007, ApJ, 655, 528

Biller, B. A., Liu, M. C., Rice, K., et al. 2015, MNRAS, 450, 4446

Boccaletti, A., Thalmann, C., Lagrange, A.-M., et al. 2015, Natur, 526, 230 Brandeker, A., Liseau, R., Olofsson, G., \& Fridlund, M. 2004, A\&A, 413, 681 Brandenburg, A., \& Dobler, W. 2002, CoPhC, 147, 471 
Burns, J. A., Lamy, P. L., \& Soter, S. 1979, Icar, 40, 1

Cameron, A. G. W., \& Pine, M. R. 1973, Icar, 18, 377

Chiang, E., \& Fung, J. 2017, ApJ, 848, 4

Currie, T., Lisse, C. M., Kuchner, M., et al. 2015, ApJL, 807, L7

Debes, J. H., Jang-Condell, H., Weinberger, A. J., Roberge, A., \& Schneider, G. 2013, ApJ, 771, 45

Dipierro, G., \& Laibe, G. 2017, MNRAS, 469, 1932

Dohnanyi, J. S. 1969, JGR, 74, 2531

Dong, R., \& Dawson, R. 2016, ApJ, 825, 77

Dong, R., \& Fung, J. 2017, ApJ, 835, 146

Dong, R., Li, S., Chiang, E., \& Li, H. 2017, ApJ, 843, 127

Eastwood, J. W., \& Hockney, R. W. 1974, JCoPh, 16, 342

Feldt, M., Olofsson, J., Boccaletti, A., et al. 2017, A\&A, 601, A7

Follette, K. B., Grady, C. A., Swearingen, J. R., et al. 2015, ApJ, 798, 132

Follette, K. B., Rameau, J., Dong, R., et al. 2017, AJ, 153, 264

Grady, C. A., Muto, T., Hashimoto, J., et al. 2013, ApJ, 762, 48

Hughes, A. M., Lieman-Sifry, J., Flaherty, K. M., et al. 2017, ApJ, 839, 86

Kalas, P., Graham, J. R., Chiang, E., et al. 2008, Sci, 322, 1345

Klahr, H., \& Lin, D. N. C. 2005, ApJ, 632, 1113

Kral, Q., \& Latter, H. 2016, MNRAS, 461, 1614

Kral, Q., Thébault, P., Augereau, J.-C., Boccaletti, A., \& Charnoz, S. 2015 , A\&A, 573, A39

Kral, Q., Wyatt, M., Carswell, R. F., et al. 2016, MNRAS, 461, 845

Krauss, O., \& Wurm, G. 2005, ApJ, 630, 1088

Krivov, A. V. 2010, RAA, 10, 383
Krivov, A. V., Herrmann, F., Brandeker, A., \& Thébault, P. 2009, A\&A, 507, 1503

Kuchner, M. J., \& Holman, M. J. 2003, ApJ, 588, 1110

Lovelace, R. V. E., Li, H., Colgate, S. A., \& Nelson, A. F. 1999, ApJ, 513, 805

Lyra, W., Johansen, A., Klahr, H., \& Piskunov, N. 2008, A\&A, 479, 883

Lyra, W., \& Kuchner, M. 2013, Natur, 499, 184

Lyra, W., McNally, C. P., Heinemann, T., \& Masset, F. 2017, AJ, 154, 146

McNally, C. P., \& Hubbard, A. 2015, ApJ, 814, 37

McNally, C. P., Lyra, W., \& Passy, J.-C. 2012, ApJS, 201, 18

Milli, J., Vigan, A., Mouillet, D., et al. 2017, A\&A, 599, A108

Nesvold, E. R., \& Kuchner, M. J. 2015, ApJ, 798, 83

Perrot, C., Boccaletti, A., Pantin, E., et al. 2016, A\&A, 590, L7

Richert, A. J. W., Lyra, W., Boley, A., Mac Low, M.-M., \& Turner, N. 2015, ApJ, 804, 95

Sezestre, É., Augereau, J.-C., Boccaletti, A., \& Thébault, P. 2017, A\&A, 607, A65

Shakura, N. I., \& Sunyaev, R. A. 1973, A\&A, 24, 337

Strubbe, L. E., \& Chiang, E. I. 2006, ApJ, 648, 652

Thébault, P., \& Augereau, J.-C. 2005, A\&A, 437, 141

van Boekel, R., Henning, T., Menu, J., et al. 2017, ApJ, 837, 132

van der Marel, N., van Dishoeck, E. F., Bruderer, S., et al. 2013, Sci, 340, 1199

Wahhaj, Z., Liu, M. C., Biller, B. A., et al. 2014, A\&A, 567, A34

Weidenschilling, S. J. 1977, Ap\&SS, 51, 153

Xie, J.-W., Brandeker, A., \& Wu, Y. 2013, ApJ, 762, 114 